\title{
Case report: EGFR-mutant lung adenocarcinoma with the TP53 and RB1 mutations showed resistance to TKI therapy
}

\author{
Yiyu Lu ${ }^{1,2 \#}$, Zhihua $\mathrm{Li}^{1,2 \#}$, Huawei Zhu ${ }^{3}$, Juan $\mathrm{Zhao}^{4}$, Mian $\mathrm{Xu}^{4}$, Weiguang $\mathrm{Gu}^{1,2}$ \\ ${ }^{1}$ Oncology Department, Nanhai People's Hospital, Foshan, China; ${ }^{2}$ The Second School of Clinical Medicine, Southern Medical University, \\ Guangzhou, China; ${ }^{3}$ Department of Oncology, Second Affiliated Hospital of Guangxi Medical University, Nanning, China; ${ }^{4}$ Shanghai OrigiMed \\ Co., Ltd., Shanghai, China \\ \#These authors contributed equally to this work. \\ Correspondence to: Weiguang Gu. Nanhai People’s Hospital, 40th Foping Road, Nanhai District, Foshan 528200, China. Email: rhp2001@21cn.com.
}

\begin{abstract}
Epidermal growth factor receptor-tyrosine kinase inhibitors (EGFR-TKIs) are a standard treatment for patients with advanced non-small-cell lung cancer (NSCLC) harboring classic EGFR mutations. However, resistance to TKIs remains a major clinical challenge. The transformation from adenocarcinoma to small-cell lung cancer (SCLC) is a rare resistance mechanism to EGFR-TKIs. In this article, we report on 2 lung adenocarcinoma patients with EGFR mutations who developed EGFRTKI resistance. In case one, the patient was initially diagnosed as lung adenocarcinoma with $E G F R$ L858R, RB1 R445*, and TP53 Y205C mutations. EGFR-TKI failed to bring satisfactory curative effect with the emergence of EGFR T790M mutation and MET amplification and finally passed away. In case two, the patient was diagnosed with lung cancer harboring EGFR L747 and TP53 R342* mutations, and EGFR-TKIs brought a progression-free survival for nine months. However, EGFR-TKI resistance was acquired, and adenocarcinoma transformed into a complex of neuroendocrine carcinoma, SCLC, and lung adenocarcinoma, with the emergence of the EGFR L747, TP53 R342*, and RB1 mutations. Follow-up treatments failed to prevent tumor progression, and the patient died These 2 cases expand our understanding of EGFR-TKI resistance, SCLC transformation, and highlight the importance of histopathology and molecular characteristics for therapeutic strategies for transformed SCLC patients.
\end{abstract}

Keywords: Adenocarcinoma; epidermal growth factor receptor-tyrosine kinase inhibitor resistance (EGFR-TKI resistance); transformed small-cell lung cancer (transformed SCLC); TP53 and RB1 mutations; case report

Submitted Jul 02, 2021. Accepted for publication Aug 12, 2021.

doi: 10.21037/apm-21-2016

View this article at: https://dx.doi.org/10.21037/apm-21-2016

\section{Introduction}

Lung cancer is the most common cause of cancerrelated death worldwide and is classified into 2 broad histological subtypes: non-small-cell lung cancer (NSCLC) and small-cell lung cancer (SCLC) $(1,2)$. Over the past decades, genomic alterations in cancer driver genes have been identified in NSCLC, and targeted therapies have become the standard care for NSCLC patients. Epidermal growth factor receptor $(E G F R)$ gene mutations can be detected in approximately $40-50 \%$ of Chinese patients with NSCLC (3), and EGFR tyrosine kinase inhibitors
(TKIs) therapy has been recommended as the first-line treatment for EGFR-mutated advanced NSCLC (4). Many patients respond well to EGFR-TKIs and enter prolonged remission; however, some experience disease progression due to acquired resistance. Several mechanisms of acquired resistance to EGFR-TKIs have been identified, such as the acquired T790M mutation, which is the primary mechanism of resistance to first-generation EGFR-TKIs, mesenchymal epithelial transition factor receptor (MET) amplification, the phosphatidylinositol-4,5-bisphosphate 3-kinase catalytic subunit alpha (PIK3CA) mutation, the B-Raf proto-oncogene $(B R A F)$ mutation, and the 

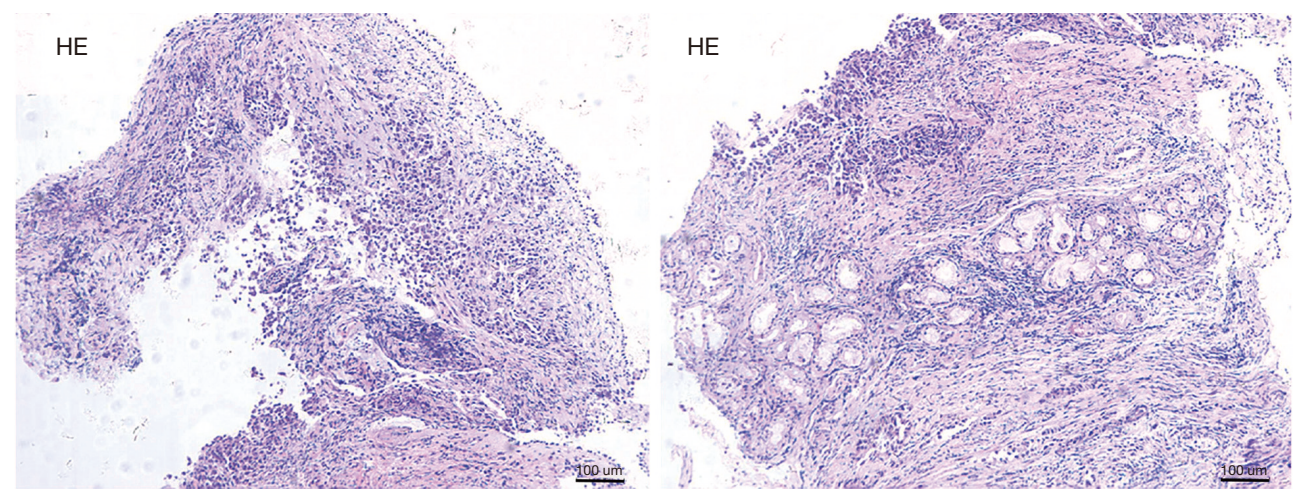

Figure 1 Lung and liver specimens from Case 1. Photomicrograph of lung biopsy with H\&E staining. Immunohistochemical analysis showing tumor cells positive for synaptophysin (+) and napsin-A (+).

transformation of adenocarcinoma with high-grade neuroendocrine carcinoma to large cell neuroendocrine carcinoma (LCNEC), SCLC, and their combined type (5). Studies have shown that TP5 3 inactivation and retinoblastoma protein ( $R B 1)$ loss might be the potential mechanisms underlying SCLC phenotype conversion after TKI resistance (6). However, the prognosis after SCLC diagnosis remains poor and current treatment strategies derived from primary SCLC seem to be ineffective.

In this article, we present 2 cases of patients who were initially diagnosed with lung adenocarcinoma harboring the EGFR mutation. Following the emergence of EGFR-TKI resistance, the TP53 and $R B 1$ mutations were revealed, and a transformation from lung adenocarcinoma to SCLC was observed in 1 patient. Such findings indicate the limited benefit of EGFR-TKIs to patients with the EGFR, TP53, and $R B 1$ methanations. We present the following article in accordance with the CARE reporting checklist (available at https://dx.doi.org/10.21037/apm-21-2016).

\section{Case presentation}

\section{Case 1}

A 57-year-old man who had never smoked presented at our hospital with cough and fever in 2016. His performance status was 0 at diagnosis. A computed tomography (CT) scan of the chest revealed a mass in the dorsal segment and posterior basal segment and effusion in the left thoracic cavity. Positron emission tomography (PET)/CT scans further revealed the metastasis of multiple lymph nodes in the bilateral subclavian fossa, mediastinum, the left lung hilar and para-aortic lymph nodes, and the liver (S6).
The lung biopsy pathology result showed that the tumor cells were positive for carcinoembryonic antigen (CEA), cytokeratin (CK)18, CK7, epithelial membrane antigen, napsin A, synaptophysin, thyroid transcription factor 1 , and Ki-67 (80\%), but negative for chromogranin A, CK14, CK20, CK5/6, tumor protein (p)40, and p63 (see Figure 1). This patient was diagnosed with poorly differentiated lung adenocarcinoma with moderately differentiated neuroendocrine tumors. The clinical tumor $(\mathrm{T})$, nodes $(\mathrm{N})$, and metastases $(\mathrm{M})$ stage was T2bN2M1b, stage IV. To determine the potential therapeutic regimens, a tumor sample was sent for a next-generation sequencing (NGS) analysis using a panel of 450 cancer-related genes. The results revealed the EGFR $\mathrm{L} 858 \mathrm{R}, R B 1 \mathrm{R} 445^{*}$, and TP53 $\mathrm{Y} 205 \mathrm{C}$ mutations. Informed consent was obtained from the patient.

The timeline of the progress of the disease is shown in Figure 2. In April 2016, the patient underwent 6 cycles of pemetrexed $(800 \mathrm{mg})$ plus cisplatin $(400 \mathrm{mg})$ chemotherapy. 6 months later, a PET/CT scan showed a good partial response (PR). During re-examination in January 2017, a CT scan showed an enlargement of the mass in the left lower lobe and a doubling of the extent of lung metastasis, suggesting progressive disease (PD). The patient was then treated with erlotinib (150 $\mathrm{mg}$ once daily) targeting the EGFR L858R mutation. However, after 3 months, CT scan showed new lesions in the liver (S6). A blood sample was subjected to NGS analysis in April 2017, which revealed an EGFR T790M mutation in addition to the existing EGFR L858R, RB1 R445*, and TP53 Y205C mutations. Osimertinib therapy ( $80 \mathrm{mg}$ once daily) was commenced immediately; however, the disease progressed rapidly, with enlarged lesions presenting in the lung and liver. The 


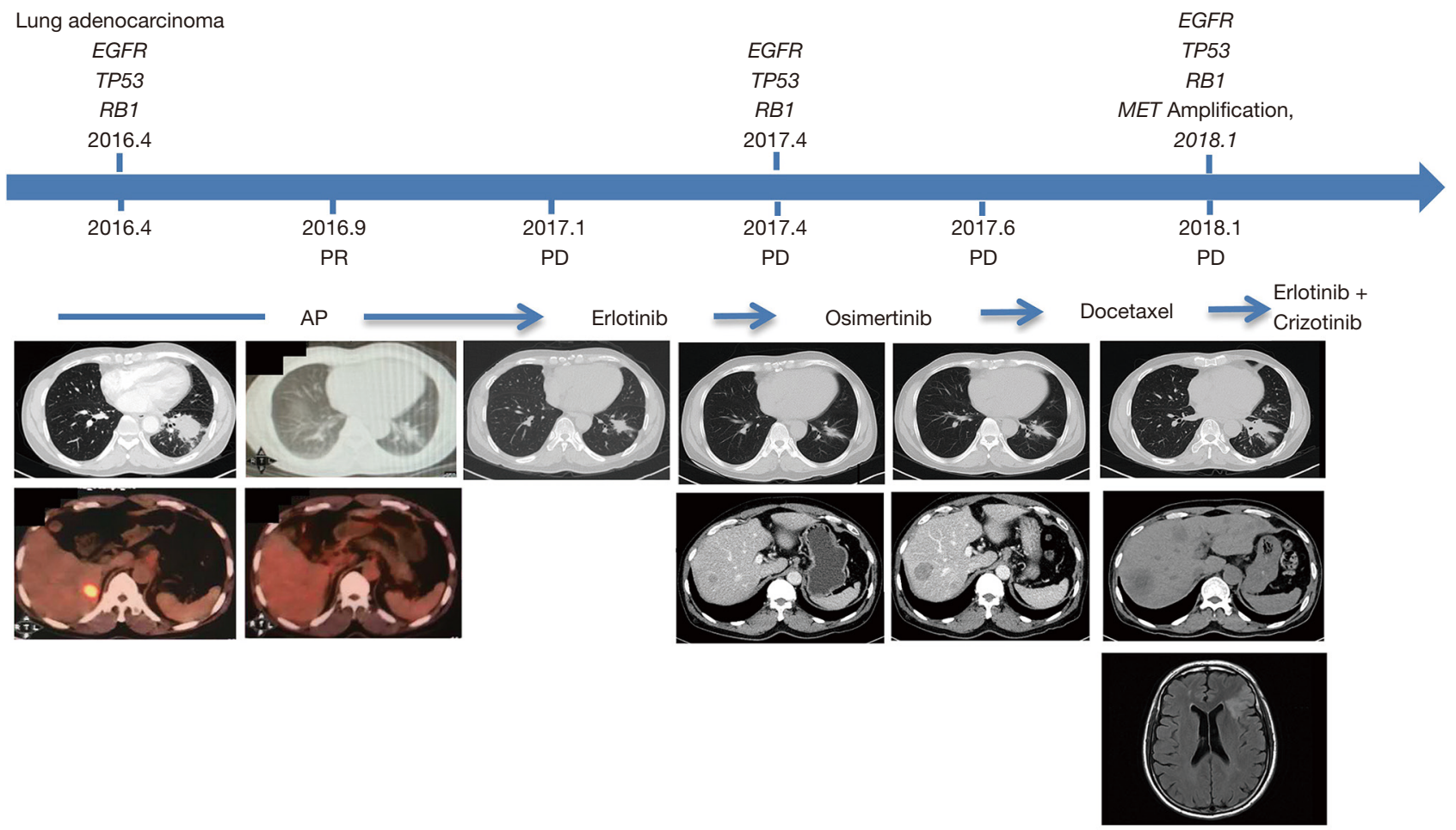

Figure 2 The treatment history of Case 1. NGS results, medical information, and a CT scan are shown. NGS, next-generation sequencing.

patient was then treated with docetaxel monotherapy, but the disease progressed, and multiple brain metastases and enlarged lesions in the lung and liver were observed. A liver biopsy showed metastases of lung adenocarcinoma, and an NGS analysis of the liver specimen showed MET amplification in addition to the existing EGFR L858R, $T P 53$, and RB1 mutations. The patient was then treated with crizotinib (250 mg once daily) plus erlotinib (125 mg once daily). Unfortunately, he died 1 month after starting this therapy.

\section{Case 2}

A 66-year-old female patient underwent surgery 6 years ago for a benign bone tumor of the left lower extremity. On April 05, 2018, she attended another hospital for treatment due to repeated fever. A chest CT scan showed a left lung mass with pleural effusion, and after a bronchoscopy pathological examination, she was diagnosed with lung cancer. The patient was treated with oral Chinese medicine (and did not receive standard treatment). On August 2, 2018, the patient was admitted to our hospital, as she had been experiencing left lower limb pain for more than
1 month. Thoracic and abdominal CT scans showed a hilus pulmonis mass in the inferior lobe of the left lung, multiple pulmonary nodules, an enlarged lymph node in the left hilus pulmonis, and a bone change in the left iliac crest and left 6th anterior rib, indicating a high possibility of lung cancer with metastasis (see Figure 3). A pathological examination of the bronchoscopy on August 3, 2018 revealed moderately differentiated lung adenocarcinoma at T4N1M1 stage IV (see Figure 4). The patient's immunohistochemistry were as follows: TTF-1(+), Napsin-A(+), CK7(+), CK5/6(-). On August 7, 2018, whole-body bone imaging showed abnormal bone metabolism in the left 7 th rib, the left iliac wing, and medial iliac crest, and the upper right femur, indicating bone metastasis.

On August 3, 2018, the lesion in the inferior lobe of the left lung was examined using NGS, and EGFR L747 and TP53 R342* mutations were detected. To target the EGFR mutation, the patient began oral icotinib (125 mg, tid) treatment on August 20, 2018, after which the lesions continued to shrink, and the efficacy evaluation was PR. The results of a NGS analysis of a blood sample on January 2, 2019 showed that the EGFR L747 mutation had disappeared, which indicated that the EGFR-TKI 

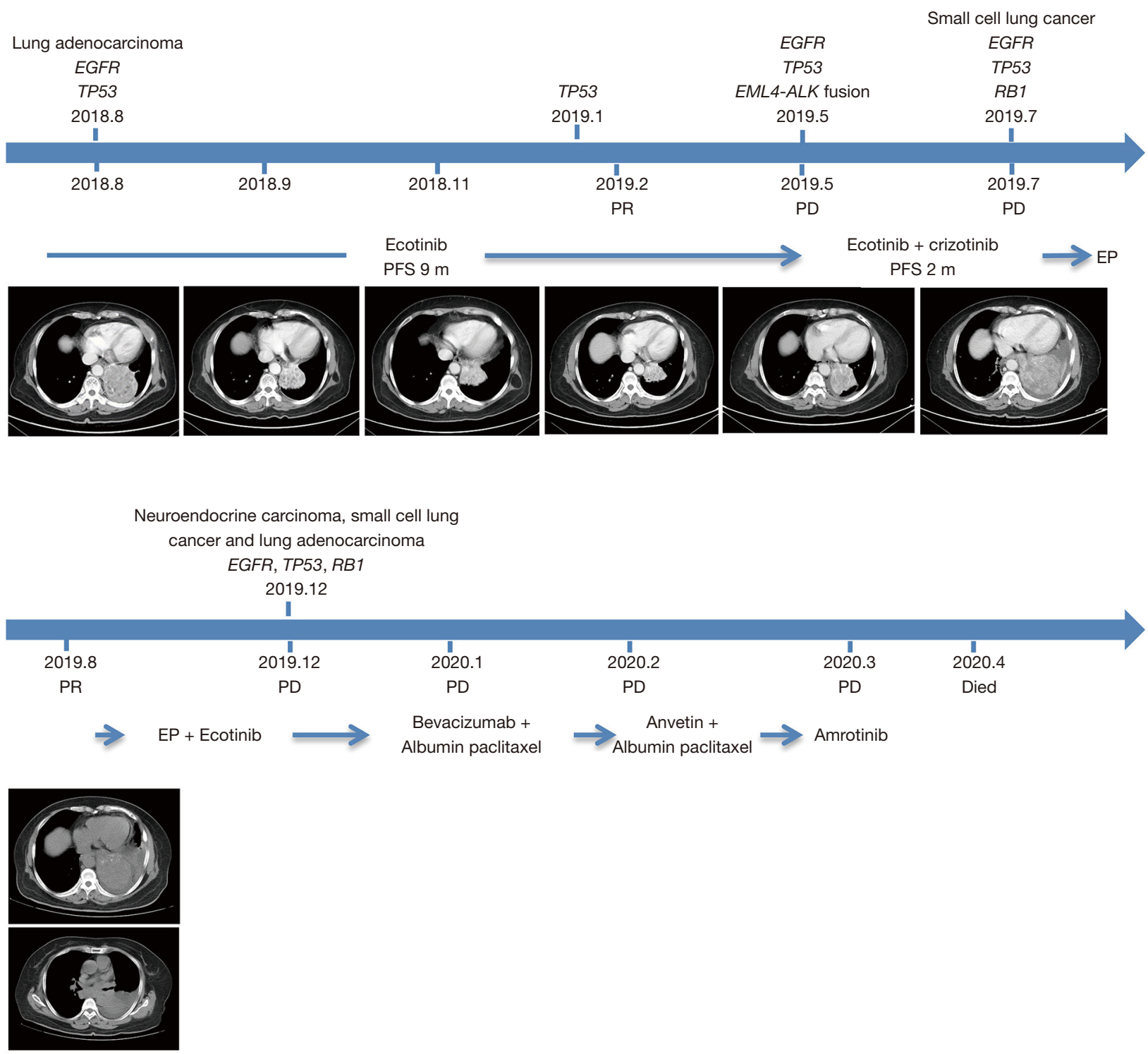

Figure 3 The treatment history of Case 2. NGS results, medical information, and a CT scan are shown. NGS, next-generation sequencing.

treatment of had been effective. However, upon being re-examined on May 21, 2019, an enlarged tumor in the inferior lobe of the left lung and lymph node enlargement in the right hilum and mediastinum were observed (see Figure 3), and the efficacy evaluation was progressed disease (PD). On May 21, 2019, another NGS analysis of a blood sample was conducted, and EML4 exon6-ALK EXON20 fusion, EGFR L747, PIK3CA, TP53 R342*, and $R B 1$ mutations were revealed. Oral icotinib $(125 \mathrm{mg}$, qd) plus crizotinib (200 $\mathrm{mg}$, bid) was administered from June 6, 2019 to July 22, 2019. However, a CT re-examination showed increased tumor volume and lymph node, demonstrating PD.

On July 23, 2019, left lung tissue was taken for a puncture biopsy and the patient was diagnosed with SCLC (see Figure 4). The immunohistochemical results showed CK(+), CK7(-), TTF-1(+), Syn(+), CD56(-), CgA(+), and $\mathrm{Ki}-67(>95 \%)$. The puncture biopsy tissue was also sent for a NGS analysis, and EGFR L747, TP53, and RB1 mutations were found. On July 27, 2019, 1 course of EP chemotherapy (etoposide $0.1 \mathrm{~g} \mathrm{~d} 1-3+$ cisplatin $30 \mathrm{mg}$ $\mathrm{d} 1-2)$ was started, and the patient's condition improved 


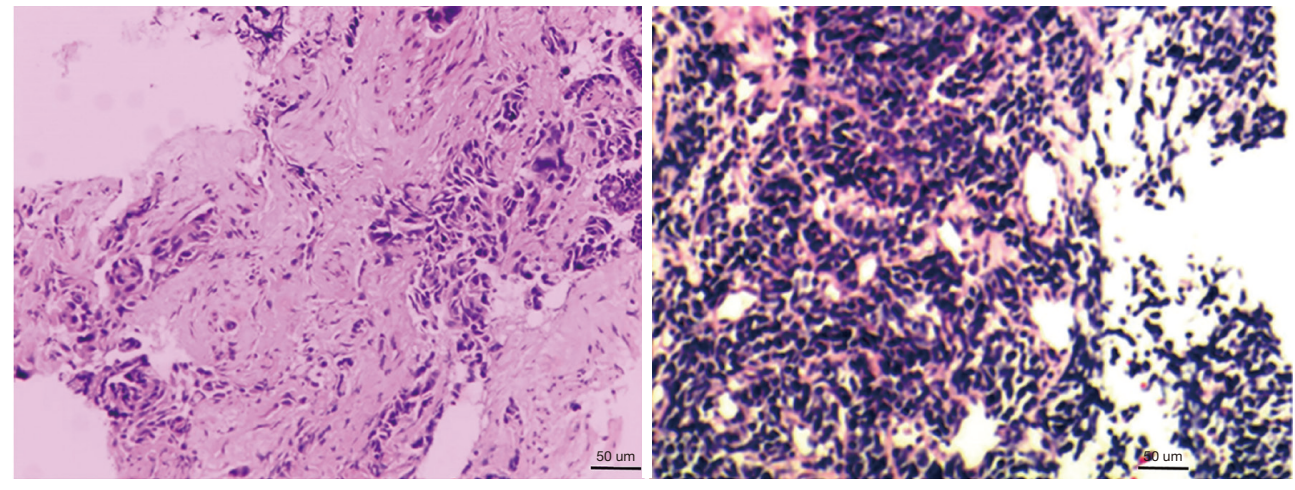

Figure 4 H\&E staining of bronchoscopy on August 3, 2018 showed lung adenocarcinoma, and H\&E staining of the left lung tissue on July 23, 2019 revealed SCLC. SCLC, small-cell lung cancer.

and the chemotherapy was well tolerated. Due to the existence of the EGFR mutation, EP+EGFR-TKI therapy was subsequently applied. A chest CT re-examination on August 5, 2019 showed that the tumor size was smaller than before. From September 2019 to December 2019, 4 courses of EP+EGFR-TKI were continued, and the re-examination in November 2019 indicated stable disease (SD). However, PD was observed.

On December 23, 2019, a puncture biopsy of the left lung mass was performed, which showed a complex of neuroendocrine carcinoma, SCLC, and lung adenocarcinoma, and a NGS analysis was performed, which revealed EGFR L747, TP53 R342*, and RB1 mutations. Icotinib treatment was stopped. On January 7, 2020, bevacizumab $(400 \mathrm{mg})$ plus albumin paclitaxel $(400 \mathrm{mg}$ ) were administered, and on February 18, 2020, anvetin plus albumin paclitaxel were administered for 1 course. A reexamination in March 2020 showed PD, and oral anlotinib (12 mg) treatment began on March 20, 2020. Unfortunately, the patient died in April 2020.

All procedures performed in studies involving human participants were in accordance with the ethical standards of the institutional and/or national research committee(s) and with the Helsinki Declaration (as revised in 2013). Written informed consent was obtained from the patients for publication of this case report and accompanying images. A copy of the written consent is available for review by the editorial office of this journal.

\section{Discussion}

The transformation of NSCLC to SCLC is observed in approximately $3-14 \%$ of EGFR0-mutated NSCLC patients with acquired EGFR-TKI resistance (7), and the comutation of RB1 and TP53 is virtually a universal feature of SCLC. In Case 2, the patient was initially diagnosed with lung adenocarcinoma with EGFR and TP53 mutations. After the EGFR-TKI (icotinib) therapy, the EGFR mutation disappeared, and PR was achieved with progression free survival (PFS) for 9 months. However, PD was observed and the patient was diagnosed with SCLC with EGFR, $T P 53$, and RB1 mutations. In relation to the mechanism of transformation in this case, it may be that both lung adenocarcinoma and SCLC were present at the initial diagnosis due to the tumor heterogeneity; however, the adenocarcinoma was only revealed by the pathological biopsy. After EGFR-TKI therapy, the adenocarcinoma cells were successfully killed, and the SCLC component survived and became dominant (8). Additionally, the emergence of the TP53 and RB1 mutations are likely to be an important driver of SCLC transformation, given that TP53 inactivation and RB1 loss are common in transformed SCLC (9).

In Case 1, the patient was initially diagnosed with lung adenocarcinoma with neuroendocrine differentiation harboring the EGFR, TP5, and RB1 mutations. NSCLC with uncertain neuroendocrine differentiation (3\%) can be further classified as LCNEC (3\%) (10), which harbors components of adenocarcinoma, squamous cell carcinoma, giant cell carcinoma, and spindle cell carcinoma (11). In another study, 45 paired samples of tumor and normal tissue from LCNEC patients were subjected to the targeted sequencing of 241 cancer-related genes, and divided into the following 3 subsets: (I) the SCLC-like subset, which was characterized by co-altered TP53 and RB1; (II) the NSCLC-like subset, which was characterized by lack of co- 
altered TP53 and RB1; and (III) the carcinoid-like subset, which was characterized by the mutations of STK11, KRAS, KEAP1, and NFE2L2 (12). In addition, Miyoshi et al. (13) observed inactivating mutations in TP53 (71\%) and $R B 1$ (26\%) in samples from 78 LCNEC patients. Based on the molecular characteristics, our patient in Case 1 is more similar to the LCNEC-SCLC-like subset than the other subsets. It may be that in the middle or late stage, the disease is very likely to transform into SCLC or mixed lung cancer. However, as only limited tissue was available for biopsy, further differential diagnoses could not be performed.

Transformed SCLC has many of the characteristics of classical SCLC (14). The most effective treatment strategies of transformed SCLC should refer to the standard treatment of classic SCLC. As SCLCs with EGFR mutations were reported to be sensitive to EGFR-TKIs (15), it is speculated that EGFR-TKIs may also be effective in treating NSCLC-transformed SCLC. Chen et al. reported on a patient with adenocarcinoma that transformed to SCLC after treatment with a 3 rd-generation-TKI for 4.3 months. After developing a resistance to EGFRTKI, the patient was treated with a variety of regimens, including etoposide combined with carboplatin (EC), irinotecan combined with oxaliplatin (IO), Abraxane, and Apatinib. However, quick progression was observed (16). Lai et al. presented 2 cases in which the patients diagnoses transformed to SCLC after 1st- and 3rd-generation EGFRTKI resistance and response to EP regimen and erlotinib; these patients passed away due to acute pulmonary embolism or severe pneumonia (17).

A recent study demonstrated that the median PFS (mPFS) for $1 \mathrm{st}-/ 2 \mathrm{nd}$-generation TKIs in NSCLC patients with EGFR mutations was 14.0 months, and the most common mutations identified in samples with transformation to SCLC were TP53 (17/25, 68.0\%), RB1 $(9 / 25,36.0 \%)$, and PIK3CA (3/25, 12.0\%). After SCLC transformation, platinum-etoposide was the most common treatment regimen. The earlier occurrence of SCLC transformation after EGFR-TKI resistance was associated with poorer prognosis (18). Similarly, in Case 1, the patient was not responsive to erlotinib or osimertinib even in the presence of L858R and T790M mutations. $M E T$ amplification, which has long been known to be an important mechanism of resistance to EGFR-TKI therapy in NSCLC, was later identified in the liver specimen. Combining crizotinib with EGFR-TKI may achieve a better clinical response (19). However, this patient was also resistant to crizotinib plus erlotinib therapy. The patient achieved PR after paclitaxel-cisplatin chemotherapy treatment, but died 1 month later. In Case 2, the patient achieved PR after icotinib and had a PFS period of 9 months. However, EGFR-TKI resistance was observed and SCLC transformation was identified. Subsequent treatment, including EP, EP + icotinib, bevacizumab + albumin paclitaxel, anvetin + albumin paclitaxel, and anlotinib, did not have good efficacy.

In summary, we reported on the cases of 2 patients with lung adenocarcinoma with EGFR mutations who developed a resistance to $E G F R$-TKI. One patient transformed to SCLC after EGFR-TKI resistance and additional TP53, and $R B 1$ mutations were identified. Subsequent treatments did not have satisfactory efficacy, and the patients' prognoses were poor. Repeated biopsies and broader molecular analyses, such as NGS, are necessary to identify changes in the histological type and describe the genetic characteristics of intricate cancers, after which appropriate treatment strategies can be identified.

\section{Acknowledgments}

We would like to thank the patients for providing samples for this study.

Funding: This research was supported by the Foshan Science and Technology Innovation Project (Medical Science and Technology Innovation Platform) (Grant No.: FS0AA-KJ218-1301-0036).

\section{Footnote}

Reporting Checklist: The authors have completed the CARE reporting checklist. Available at https://dx.doi. org/10.21037/apm-21-2016

Conflicts of Interest: All authors have completed the ICMJE uniform disclosure form (available at https://dx.doi. org/10.21037/apm-21-2016). JZ and MX received personal fees from Shanghai OrigiMed Co., Ltd. The other authors have no conflicts of interest to declare.

Ethical Statement: The authors are accountable for all aspects of the work in ensuring that questions related to the accuracy or integrity of any part of the work are appropriately investigated and resolved. This study 
was approved by the Ethics Committee of the Nanhai People's Hospital. All procedures performed in studies involving human participants were in accordance with the ethical standards of the institutional and/or national research committee(s) and with the Helsinki Declaration (as revised in 2013). Written informed consent was obtained from the patients for publication of this case report and accompanying images. A copy of the written consent is available for review by the editorial office of this journal.

Open Access Statement: This is an Open Access article distributed in accordance with the Creative Commons Attribution-NonCommercial-NoDerivs 4.0 International License (CC BY-NC-ND 4.0), which permits the noncommercial replication and distribution of the article with the strict proviso that no changes or edits are made and the original work is properly cited (including links to both the formal publication through the relevant DOI and the license). See: https://creativecommons.org/licenses/by-nc$\mathrm{nd} / 4.0 \%$.

\section{References}

1. Molina JR, Yang P, Cassivi SD, et al. Non-small cell lung cancer: epidemiology, risk factors, treatment, and survivorship. Mayo Clin Proc 2008;83:584-94.

2. Bray F, Ferlay J, Soerjomataram I, et al. Global cancer statistics 2018: GLOBOCAN estimates of incidence and mortality worldwide for 36 cancers in 185 countries. CA Cancer J Clin 2018;68:394-424.

3. Wen S, Dai L, Wang L, et al. Genomic Signature of Driver Genes Identified by Target Next-Generation Sequencing in Chinese Non-Small Cell Lung Cancer. Oncologist 2019;24:e1070-e1081.

4. Hsiue EH, Lee JH, Lin CC, et al. Safety of gefitinib in non-small cell lung cancer treatment. Expert Opin Drug Saf 2016;15:993-1000.

5. Piotrowska Z, Sequist LV. Treatment of EGFR-Mutant Lung Cancers After Progression in Patients Receiving First-Line EGFR Tyrosine Kinase Inhibitors : A Review. JAMA Oncol 2016;2:948-54.

6. Roca E, Gurizzan C, Amoroso V, et al. Outcome of patients with lung adenocarcinoma with transformation to small-cell lung cancer following tyrosine kinase inhibitors treatment: A systematic review and pooled analysis. Cancer
Treat Rev 2017;59:117-22.

7. Tan CS, Gilligan D, Pacey S. Treatment approaches for EGFR-inhibitor-resistant patients with non-small-cell lung cancer. Lancet Oncol 2015;16:e447-e459.

8. Yano S, Wang W, Li Q, et al. Hepatocyte growth factor induces gefitinib resistance of lung adenocarcinoma with epidermal growth factor receptor-activating mutations. Cancer Res 2008;68:9479-87.

9. Lee JK, Lee J, Kim S, et al. Clonal History and Genetic Predictors of Transformation Into Small-Cell Carcinomas From Lung Adenocarcinomas. J Clin Oncol 2017;35:3065-74.

10. Howe MC, Chapman A, Kerr K, et al. Neuroendocrine differentiation in non-small cell lung cancer and its relation to prognosis and therapy. Histopathology 2005;46:195-201.

11. Travis WD. Lung tumours with neuroendocrine differentiation. Eur J Cancer 2009;45 Suppl 1:251-66.

12. Rekhtman N, Pietanza MC, Hellmann MD, et al. Next-Generation Sequencing of Pulmonary Large Cell Neuroendocrine Carcinoma Reveals Small Cell Carcinoma-like and Non-Small Cell Carcinoma-like Subsets. Clin Cancer Res 2016;22:3618-29.

13. Miyoshi T, Umemura S, Matsumura Y, et al. Genomic Profiling of Large-Cell Neuroendocrine Carcinoma of the Lung. Clin Cancer Res 2017;23:757-65.

14. Niederst MJ, Sequist LV, Poirier JT, et al. RB loss in resistant EGFR mutant lung adenocarcinomas that transform to small-cell lung cancer. Nat Commun 2015;6:6377.

15. Tatematsu A, Shimizu J, Murakami Y, et al. Epidermal growth factor receptor mutations in small cell lung cancer. Clin Cancer Res 2008;14:6092-6.

16. Chen S, He Y, Liu J, et al. Third-Generation TKI Resistance Due to SCLC Transformation: A Case Report and Brief Review. Onco Targets Ther 2019;12:11305-11.

17. Lai L, Meng W, Wei J, et al. Transformation of NSCLC to SCLC after 1st- and 3rd-generation EGFR-TKI resistance and response to EP regimen and erlotinib: 2 CARE-compliant case reports. Medicine (Baltimore) 2021;100:e25046.

18. Wang W, Xu C, Chen H, et al. Genomic alterations and clinical outcomes in patients with lung adenocarcinoma with transformation to small cell lung cancer after 
treatment with EGFR tyrosine kinase inhibitors: A multicenter retrospective study. Lung Cancer 2021;155:20-7.

19. Giroux-Leprieur E, Dumenil C, Chinet T. Combination of Crizotinib and Osimertinib or Erlotinib Might

Cite this article as: $\mathrm{Lu} \mathrm{Y,} \mathrm{Li} \mathrm{Z,} \mathrm{Zhu} \mathrm{H}$, Zhao J, Xu M, $\mathrm{Gu} W$. Case report: EGFR-mutant lung adenocarcinoma with the TP53 and RB1 mutations showed resistance to TKI therapy. Ann Palliat Med 2021;10(12):12886-12893. doi: 10.21037/apm-212016
Overcome MET-Mediated Resistance to EGFR Tyrosine Kinase Inhibitor in EGFR-Mutated Adenocarcinoma. J Thorac Oncol 2018;13:e232-e234.

(English Language Editor: L. Huleatt) 